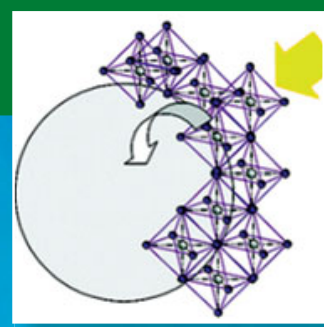

\title{
Methylammonium lead triiodide perovskite solar cells: A new paradigm in photovoltaics
}

\author{
Mohammad Khaja Nazeeruddin and Henry Snaith, Guest Editors
}

\begin{abstract}
Perovskite solar cells based on methylammonium lead triiodide witnessed unprecedented progress after the seminal work of Miyasaka and co-workers in 2009, where they employed perovskite nanocrystals as sensitizers in a dye-sensitized solar-cell configuration. After key breakthroughs with solid-state perovskite photovoltaics in 2012, research efforts have grown exponentially, and several groups have demonstrated that the perovskite concomitantly acts as a light absorber and an electron and hole transporter in both mesoscopic networks and solid polycrystalline layers, where the perovskite layer can be deposited using a broad range of techniques. The methylammonium lead triiodide perovskite bandgap has been tuned by substituting various cations and anions. By optimizing the crystalline quality of the perovskite absorber and film formation by solvent engineering, a remarkable power-conversion efficiency of over $20 \%$ has been demonstrated, highlighting the exceptional photovoltaic properties of perovskite materials. The high efficiencies are due to a combination of long carrier lifetimes, substantial charge-carrier mobilities, and remarkably benign electronic defects. This issue highlights various deposition methods of the perovskite absorber, such as single-step, sequential, dual-source sublimation, and solution and sublimation processes, as well as holetransporting-free and tandem perovskite solar cells.
\end{abstract}

\begin{abstract}
Introduction
Organic-inorganic hybrid perovskite materials pioneered by Mitzi in 2001 have been recognized for their use in optoelectronic applications. ${ }^{1}$ Group 14 iodometalates, such as $\mathrm{ABX}_{3}$, adopt perovskite structures that are flexible for compositional variations. A hybrid perovskite results when " $\mathrm{A}$ " is a small organic cation, " $\mathrm{B}$ " is a Group 14 metal lead $(\mathrm{Pb})$, and " $\mathrm{X}$ " is a halogen that forms a three-dimensional inorganic framework with the organic cation. The ease with which these hybrid perovskite materials can be prepared and processed from solution have made them attractive for photovoltaic (PV) applications following the pioneering work of Miyasaka and co-workers in 2009, where they replaced dye in dye-sensitized solar cells and obtained a power-conversion efficiency of $3.8 \%$ using iodine/triiodide-based liquid electrolyte. ${ }^{2}$ After an initial slow following, perovskite solar cells have created a tsunami effect in the PV community because of their highpower-conversion efficiencies reaching over $20 \%$, enabled by their unexpected optoelectronic properties. ${ }^{3}$
\end{abstract}

Initially, perovskite solar-cell device architecture was based on dye-sensitized solar cells, as shown in Figure 1. ${ }^{4}$ The device is composed of three major components: an absorber to absorb light, a semiconductor to accept electrons from photoexcited perovskite, and a hole-transporting $p$-type semiconductor sandwiched between two conducting electrodes. The working principle of a perovskite solar cell involves promotion of electrons from the ground state of the perovskite to the excited state upon irradiation with photons and injection of the excited electrons to the conductance band of the semiconductor. The positive charge on the perovskite is transferred to the hole-transporting material and then to the gold contact electrode. The perovskite plays a critical role in this process for determining the amount of light absorbed both in intensity and breadth (wavelength range).

The key breakthrough, which sparked broad excitement in the PV community, was the realization of $10 \%$ efficient solid-state perovskite solar cells in $2012 .{ }^{4,5}$ Specifically, the unexpectedly high open-circuit voltages generated by these 


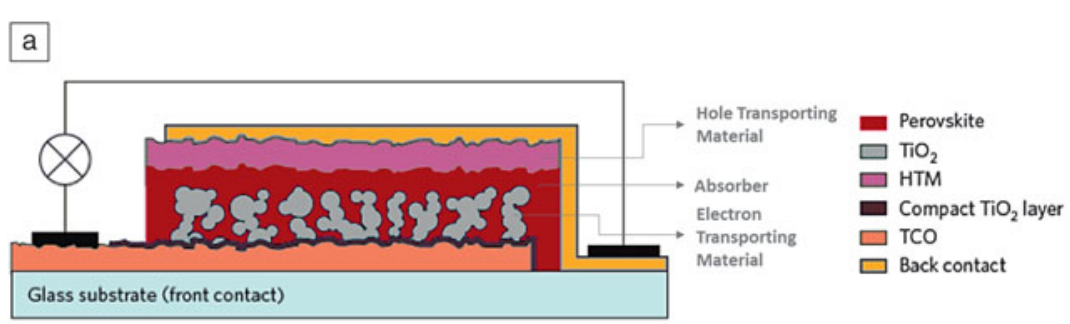

$\mathrm{b}$

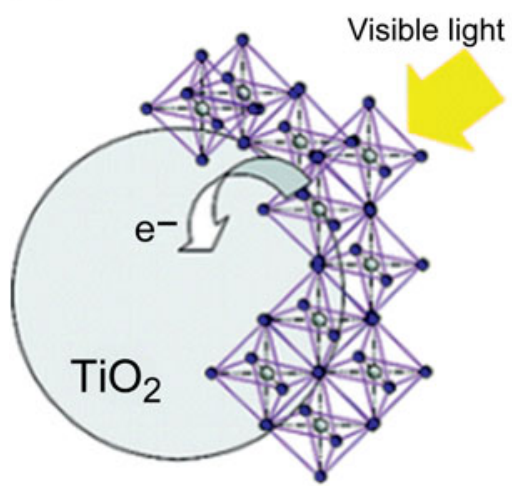

Perovskite nanocrystalline sensitizers

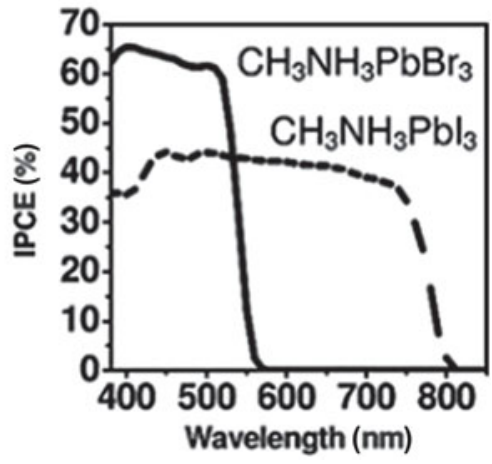

Figure 1. (a) A schematic illustration of a perovskite-sensitized solar cell. (b) $\mathrm{TiO}_{2}$ undergoing photoexcitation and electron transfer. The filled circles represent iodide, and the empty circles represent lead. (c) The incident photon-to-electron conversion efficiency (IPCE) spectra for perovskite-sensitized solar cells. Reprinted with permission from Reference 4. (C) 2012 AAAS. Note: HTM, hole transport material; MAI, methylammonium iodide; TCO, transparent conducting oxide.

layer, a 100-200-nm-thick mesoporous $\mathrm{TiO}_{2}$ layer, which acts as an electron transport material and scaffold infilled with perovskite, a solid perovskite overlayer of $200-300 \mathrm{~nm}$ thickness, an additional light-absorbing layer, a hole-transporting layer, and a metal back electrode..$^{10}$ Often, mesoporous $\mathrm{Al}_{2} \mathrm{O}_{3}$ is used in place of $\mathrm{TiO}_{2}$, or the scaffold is completely removed in planar heterojunction cells. A planar heterojunction device architecture typically consists of glass/FTO/compact metal oxide/ perovskite/hole transporter/gold contact.

The most commonly used hole-transporting materials in perovskite solar cells are spiroOMeTAD $\left(2,2^{\prime} 7,7^{\prime}\right.$-tetrakis $(N, N$-di-p-methoxyphenylamine)-9, $9^{\prime}$-spirobifluorene $)^{11}$ and PTAA (polytertiary aryl amine) polymer. ${ }^{12}$ The electronic properties of the perovskite and hole-transporting material have been extensively investigated, including the optimization of the performance by chemical $p$-doping of spiro-OMeTAD.

Optimization of the thickness of the various layers and the morphology of these layers affects overall device performance. For example, by varying the thickness of the mesoporous $\mathrm{TiO}_{2}$ layer and the perovskite overlayer, the absorption of the device can be tuned, yielding significant enhancement in the short-circuit current. A too thin perovskite overlayer (less than $200 \mathrm{~nm}$ ) results in low photocurrent due

solar cells placed their potential for high performance in the realm of highly crystalline semiconductors such as GaAs and $\mathrm{Si}$, as opposed to electronically disordered semiconductors such as organics, metal oxides, and amorphous silicon. Since these early reports, a commonality in improvements in efficiency has been improvements in crystallinity quality and smoothness and uniformity of the solid crystalline perovskite layer responsible for the light absorption and generation of free charge carriers. The variation in device architectures now occurs in the nature of the positive $(p)$ and negative $(n)$ contact materials, with the optional insertion of a thin interlayer of mesoporous scaffold to either aid perovskite film formation or charge extraction. The solar cells can thus be considered $n-i-p$ and $p-i-n$ structures, where the perovskite is an intrinsic semiconductor, and light enters through the $n$-type or the $p$-type layer, respectively. ${ }^{6-9}$

\section{Device architectures and materials Configurations}

There are many different materials and configurations that encompass current state-of-the-art perovskite solar cells, ranging from all organic to all inorganic contacts, with or without the inclusion of a mesoporous scaffold. Some of the most efficient cells reported to date comprise a fluorine-doped tin oxide-FTO-coated glass, a 20-50-nm-thick $\mathrm{TiO}_{2}$ compact to insufficient absorption. However, an excessively thick overlayer results in a lower fill factor. The fill factor is the ratio of the maximum obtainable power to the product of the open-circuit voltage and short-circuit current density.

The grain size of the perovskite crystals also plays an important role. Typically, the thickness of the hole-transporting material in perovskite solar cells is in the range of $150 \mathrm{~nm}$ to $200 \mathrm{~nm}$. If this is too thin, then it provokes short circuits, whereas if it is too thick, this decreases the fill factor. ${ }^{13}$ In fact, state-of-the-art perovskite devices that incorporate mesoporous $\mathrm{TiO}_{2}$ can be considered as a combination of a mesoporous $\mathrm{TiO}_{2} /$ perovskite bulk heterojunction layer and a flat solid perovskite layer.

There are now many research teams advancing different cell configurations in parallel with insulating or semiconducting mesoporous scaffolds and with organic or inorganic semiconductor contacts for planar heterojunctions. A single dominant architecture (or contact material and configuration) may emerge, but it also may be possible that a number of suitable perovskite cell "technologies" can emerge, similar to the handful of commercial silicon cell technologies that currently exist.

\section{Perovskite material}

The typical crystalline structure of perovskite material is $\mathrm{ABX}_{3}$, where $\mathrm{A}=\mathrm{MA}$ cation, $\mathrm{B}=$ lead di-cation, and $\mathrm{X}=$ iodide; 
this is called MA lead triiodide perovskite. The MA lead triiodide perovskite exhibits direct bandgap absorption from the top of the valence band to the bottom of the conduction band. The valence band is dominated by iodide $p$ orbitals, and the conduction band consists of $\mathrm{Pb} p$ orbitals, therefore the transitions are $p$-to- $p$ orbitals. ${ }^{14}$ The bandgap of $\mathrm{ABX}_{3}$ perovskite can be tuned by varying A, B, and X. ${ }^{15}$ Further, the bandgap can be modulated by appropriate selection of mixed cations and halogens. ${ }^{16,17}$

Among these combinations, perovskites containing MA and formamidinium (FA) as the $\mathrm{A}$ cation and metal $\mathrm{Pb}^{2+}$ as the $\mathrm{B}$ cation have raised interest due to their excellent optoelectronic properties, potential for low-temperature solution processable films, and stability. ${ }^{18,19}$ In addition, $\mathrm{ABX}_{3}$ properties are amenable to the formation and nature of the growth of perovskite materials. ${ }^{4}$ The $\mathrm{MA}$ lead iodide $\left(\mathrm{CH}_{3} \mathrm{NH}_{3} \mathrm{PbI}_{3}\right)$ perovskite material has a panchromatic absorption down to ca. $800 \mathrm{~nm}$, direct bandgap of $1.57 \mathrm{eV}$, large absorption coefficient of $1.5 \times 10^{4} \mathrm{~cm}^{-1}$ at $550 \mathrm{~nm},{ }^{2}$ low exciton binding energy $\left(<10 \mathrm{meV}\right.$ in $\left.\mathrm{MAPbI}_{3}\right),{ }^{20}$ very high charge-carrier mobility $\left(66 \mathrm{~cm}^{2} / \mathrm{Vs}\right.$ for $\left.\mathrm{MAPbI}_{3}\right),{ }^{20}$ ambipolar charge transport, ${ }^{21}$ large electron and hole diffusion lengths (over $1 \mu \mathrm{m}$ and potentially over $100 \mu \mathrm{m}),{ }^{22}$ and low nonradiative recombination rates. ${ }^{23}$

Perovskite solar cells show unreliable efficiencies due to scan speed-dependent hysteresis observed in the photocurrentvoltage curves of the cells, which could be a bottleneck in the development of photovoltaic technology based on them (Figure 2). Various studies regarding the origin of the hysteresis have been documented, such as ferroelectricity caused by dipoles of MA molecules, ${ }^{24}$ ion migration, ${ }^{25}$ and unbalanced charge collection rate at the interfaces. ${ }^{26}$ Among these, unbalanced charge collection and ion migration seem to be realistic causes for hysteresis behavior.

The most striking evidence to support the imbalanced charge extraction comes from the $p-i-n$ structures (inverted perovskite solar cell), where the hysteresis is significantly lower compared to the $n-i-p$ (normal perovskite solar cells) structures. ${ }^{27}$ The imbalanced charge collection is probably related to the electrical character of the perovskite materials and charge accumulation at the interface between perovskite and $n$-type/p-type layers. ${ }^{28}$ Therefore, the control of perovskite crystal morphology and growth, passivation of the perovskite layer by interfacial engineering, and development of new $n$ - and $p$-type materials for efficient charge extraction at the heterojunctions are paramount to further improve the powerconversion efficiency, specifically long-term stabilized power output of perovskite solar cells. Figure 2 shows an optimized perovskite solar cell (Figure 2a) and significantly reduced hysteresis (Figure 2b).

\section{Fabrication}

Besides the excellent PV performance of the organic-inorganic halide perovskite, the perovskite material is attractive because of its versatility in terms of fabrication techniques. There are several deposition methods available for perovskite films, including one-step precursor solution deposition, ${ }^{5}$ two-step sequential deposition, ${ }^{18}$ dual-source vapor deposition, ${ }^{29}$ and vapor-assisted solution procession. ${ }^{30}$

In the one-step precursor solution deposition, the MA lead iodide perovskite is deposited onto the mesoporous metal oxide film by spin coating of a precursor solution of $1: 1$ $\mathrm{CH}_{3} \mathrm{NH}_{3} \mathrm{I}$ and $\mathrm{PbI}_{2}$ in $\gamma$-butyrolactone or dimethylformamide (DMF) or a mixture of dimethyl sulfoxide and DMF solvents on top of the 200-400-nm-thick film of $\mathrm{TiO}_{2}$ (anatase) nanoparticles (Figure 3a). Burschka et al. reported a 15\% power-conversion efficiency, where the perovskite was deposited by a novel sequential deposition technique on a mesoporous $\mathrm{TiO}_{2}$ film (Figure 3b). ${ }^{18}$

In an inverted layout, similar to that employed in all organic polymeric solar cells, the perovskite layer was deposited using a dual source sublimation method and used as the electron donor in combination with an electron acceptor [6,6]-phenyl
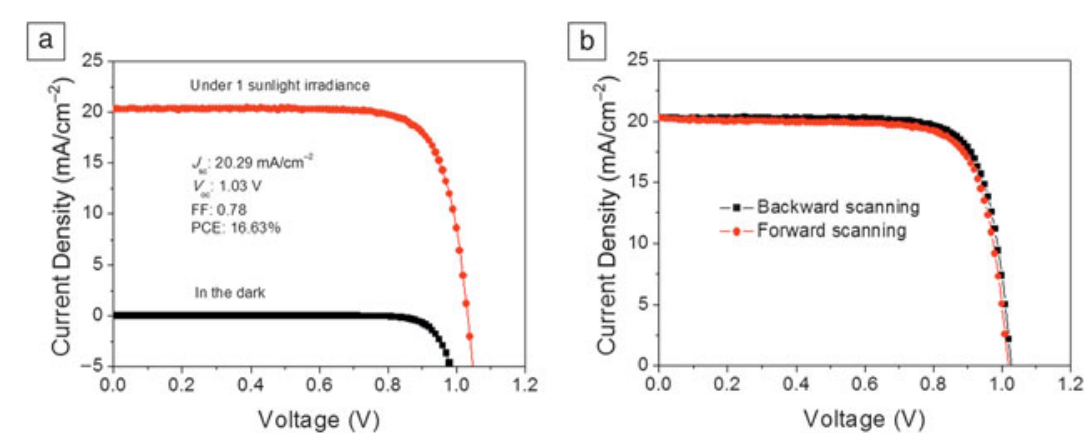

Figure 2. Representative current-voltage characteristics of the champion cell-the best performing cell to date using a methylammonium lead triiodide perovskite-(a) and current density-voltage ( $J-V$ curve hysteresis by scanning forward and backward (b). The current-voltage data in (a) show the best efficiency obtained with an optimized perovskite morphology, and the (b) $J-V$ curves at different scanning directions exhibit small hysteresis. Note: $J_{s c}$, short-circuit current density; $V_{\text {oc }}$, open-circuit voltage; FF, fill factor; PCE, power-conversion efficiency. Reprinted with permission from Reference 35. (C)2015 Wiley.
$\mathrm{C}_{61}$-butyric acid methylester PCBM (Figure $3 \mathrm{c}$ ). ${ }^{31}$ Holes are extracted via the transparent conductor poly(3,4-ethylenedioxythiophene):poly (styrenesulfonic acid) (PEDOT:PSS). In this configuration, the sublimated MA lead iodide perovskite layers are sandwiched between two very thin electron and hole blocking layers consisting of polytertiary arylamine and PCBM organic molecules. The organic materials were deposited using solutionbased processes, whereas the $\mathrm{CH}_{3} \mathrm{NH}_{3} \mathrm{PbI}_{3}$ perovskite and the metal contact were deposited using thermal evaporation under vacuum, which reached efficiencies as high as $12 \%$ at $100 \mathrm{~mW} \mathrm{~cm}^{-2}{ }^{31}$

Yang et al. reported a low-temperature approach for the deposition of the perovskite absorbing layer called vapor-assisted solution processing (VASP), which is similar to 

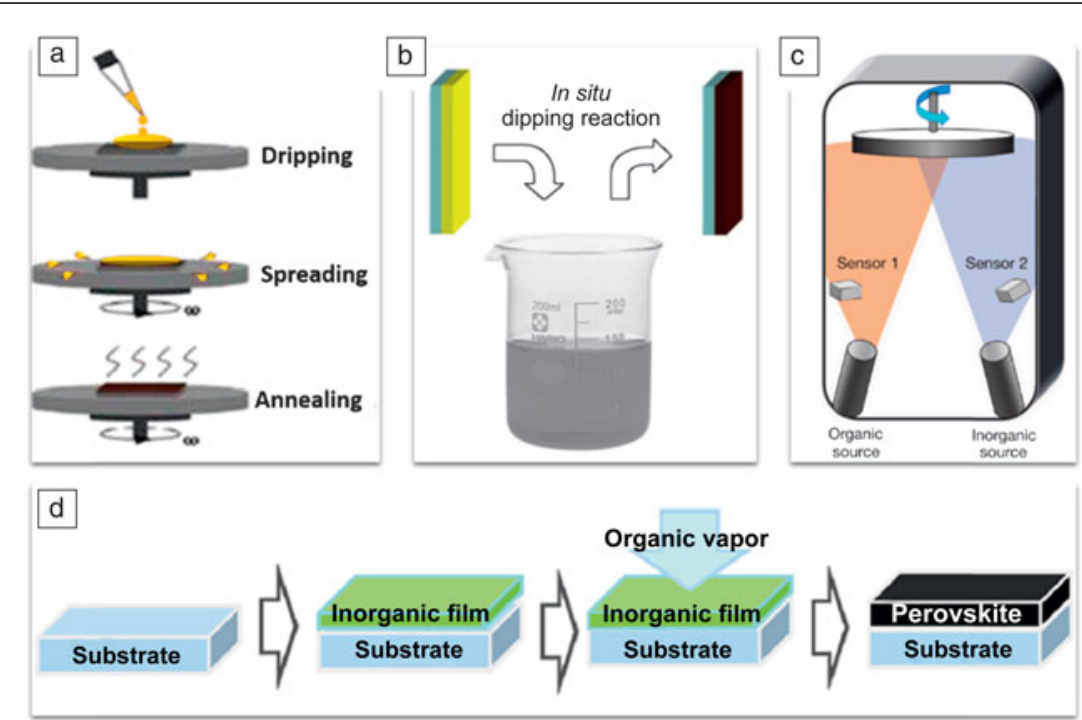

Figure 3. Four general methods to prepare perovskite active layers. (a) One-step precursor deposition; (b) sequential deposition method; (c) dual-source vapor deposition; and (d) vapor-assisted solution process. Reprinted with permission from Reference 15. (c) 2014 Royal Society of Chemistry.

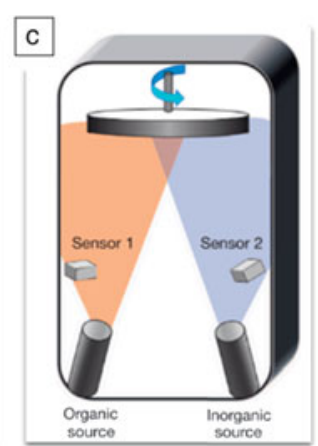

performance of the perovskite solar cells, yielding a National Renewable Energy Laboratory certified efficiency of $20.1 \%$. Lee and Park describe a two-step deposition method for a perovskite material that leads to high-efficiency perovskite solar cells. Next, Sessolo et al. show the latest advances in the vacuum deposition of perovskite films with an emphasis on PV applications. Zhou et al. discuss high-quality perovskite films deposited via the facile low-temperature $\left(<150^{\circ} \mathrm{C}\right)$ vapor-assisted solution process. Etgar covers hole-transport-material-free solar cells that could benefit future PV and optoelectronic applications. Finally, Bailie and McGehee discuss the challenges facing perovskite-based tandems in attaining efficiency values over $25 \%$ to reach commercial competitiveness.

\section{Conclusion}

Organic-inorganic metal halide perovskites, such as $\mathrm{CH}_{3} \mathrm{NH}_{3} \mathrm{PbI}_{3}$, are low-cost precursors, capable of being processed via a variety of scalable methods and delivering high power-

a combination of the sequential deposition method and dualsource vapor deposition. ${ }^{32}$ In this process, $\mathrm{PbI}_{2}$ films are deposited onto a FTO glass coated with a compact layer of $\mathrm{TiO}_{2}$, followed by annealing in methylammonium iodide vapor at $150^{\circ} \mathrm{C}$ in a $\mathrm{N}_{2}$ atmosphere for $2 \mathrm{~h}$ to form perovskite films (Figure 3d). The perovskite film derived from this approach exhibits full surface coverage, uniform grain structure with grain size of up to a few nanometers, and 100\% precursor transformation. VASP presents a simple, controllable, and versatile approach to the pursuit of high-quality perovskite films and the resulting high-performance PV devices.

Several groups have investigated charge transfer dynamics by using different wavelengths and fluorescence of femtosecond excitation of perovskite films and by probing the visiblenear-infrared transient absorptions of the generated charge carriers. If the scaffold material is a mesoporous layer of $\mathrm{TiO}_{2}$, the data show sub-picosecond electron injection into titania in $170 \mathrm{fs}$, and electron and hole relaxations in the range of $0.5-$ 0.7 ps within perovskite and titania conduction bands. ${ }^{33}$ The contribution of trap states in the recombination processes is small, thus increasing the survival probability of the charge in the excited perovskite collecting at the respective electrodes.

\section{In this issue}

In this issue of MRS Bulletin, we have invited leading experts to share their data based on one-step precursor solution deposition, two-step sequential deposition, dual-source vapor deposition, vapor-assisted solution processed techniques, and hole-transporting material free perovskite solar cells. In the first article, Noh and Seok describe perovskite deposition using a chemical engineering process that gives extremely uniform and dense perovskite layers and remarkably improved the conversion efficiencies in solar cells that are comparable to thin-film and crystalline silicon solar cells. As demonstrated, the applications of $\mathrm{CH}_{3} \mathrm{NH}_{3} \mathrm{PbI}_{3}$ beyond solar cells-lightemitting diodes, photo-detectors, light-emitting field-effect transistors, and lasers $\left(\mathrm{CH}_{3} \mathrm{NH}_{3} \mathrm{PbI}_{3}\right)$ - signify the practicality of the perovskite materials. However, there are drawbacks to perovskite materials: sensitivity toward humidity, instability under heat and light conditions, and unreliability under operating conditions. Solutions to these drawbacks are being discovered through the molecular engineering of functionalized interface materials that not only function as efficient charge extractors, but also act as a shield against humidity and UV light-induced degradation. The prospects for perovskite materials in various applications are extremely bright, with markets spanning from solar cells to sensing, imaging, environmental applications, and optoelectronics.

The techniques and data presented in this issue will stimulate readers and the materials communities to further explore the amazing properties of perovskite materials, reaching the ambitious goal of reducing our dependence on non-renewable power-generating technologies. If perovskite solar cells are proven to be a reliable technology, every house can produce its own electricity and store the surplus - or feed it back to the grid. The dream is that, eventually, the power consumer will become the producer.

\section{Acknowledgments}

The authors acknowledge funding from the European Union Seventh Framework Programme [FP7/2007-2013] under grant agreement no. 604032 of the MESO project. They thank Peng Gao in assisting with the cover art and reading the manuscript. 


\section{References}

1. D.B. Mitzi, Chem. Mater. 13, 3283 (2001).

2. A. Kojima, K. Teshima, Y. Shirai, T. Miyasaka, J. Am. Chem. Soc. 131, 6050 (2009).

3. National Renewable Energy Laboratory, Best Research-Cell Efficiencies; http://www.nrel.gov/ncpv/images/efficiency_chart.jpg.

4. M.M. Lee, J. Teuscher, T. Miyasaka, T.N. Murakami, H.J. Snaith, Science 338, 643 (2012)

5. H.S. Kim, C.R. Lee, J.H. Im, K.B. Lee, T. Moehl, A. Marchioro, S.J. Moon, R. Humphry-Baker, J.H. Yum, J.E. Moser, M. Grätzel, N.G. Park, Sci. Rep. 2, 591 (2012)

6. L. Etgar, P. Gao, Z. Xue, Q. Peng, A.K. Chandiran, B. Liu, M.K. Nazeeruddin, M. Grätzel, J. Am. Chem. Soc. 134, 17396 (2012).

7. S. Kazim, M.K. Nazeeruddin, M. Grätzel, S. Ahmad, Angew. Chem. Int. Ed. 53, $2812(2014)$

8. W.S. Yang, J.H. Noh, N.J. Jeon, Y.C. Kim, S. Ryu, J. Seo, S.I. Seok, Science 348, 1234 (2015).

9. J.-H. Heo, H.J. Han, D. Kim, T. Ahn, S.H. Im, Energy Environ. Sci. 8, 1602 (2015). 10. N.J. Jeon, J.H. Noh, W.S. Yang, Y.C. Kim, S. Ryu, J. Seo, S.I. Seok, Nature 517, $476(2015)$

11. J.-H. Im, I.-H. Jang, N. Pellet, M. Grätzel, N.-G. Park, Nat. Nanotechnol. 2, 705 (2014)

12. N.J. Jeon, J.H. Noh, Y.C. Kim, W.S. Yang, S. Ryu, S.I. Seok, Nat. Mater. 13, 897 (2014).

13. W. Nie, H. Tsai, R. Asadpour, J. Blancon, A.J. Neukirch, G. Gupta, J.J. Crochet, M. Chhowalla, S. Tretiak, M.A. Alam, H. Wang, A.D. Mohite, Science 347, 522 (2015). 14. N.-G. Park, J. Phys. Chem. Lett. 4, 2423 (2013).

15. P. Gao, M. Grätzel, M.K. Nazeeruddin, Energy Environ. Sci. 7, 2448 (2014). 16. N. Pellet, P. Gao, G. Gregori, T.-Y.Y. Yang, M.K. Nazeeruddin, J. Maier, M. Grätzel, Angew. Chem. Int. Ed. 53, 3151 (2014).

17. J.H. Noh, S.H. Im, J.H. Heo, T.N. Mandal, S.I. Seok, Nano Lett. 13, 1764 (2013). 18. J. Burschka, N. Pellet, S.-J. Moon, R. Humphry-Baker, P. Gao, M.K. Nazeeruddin, M. Grätzel, Nature 499, 316 (2013).
19. S. Pang, H. Hu, J. Zhang, S. Lv, Y. Yu, F. Wei, T. Qin, H. Xu, Z. Liu, G. Cui, Chem. Mater. 26, 1485 (2014)

20. T.J. Savenije, C.S. Ponseca, L. Kunneman, M. Abdellah, K. Zheng, Y. Tian, Q. Zhu, S.E. Canton, I.G. Scheblykin, T. Pullerits, A. Yartsev, V. Sundström, J. Phys. Chem. Lett. 5, 2189 (2014).

21. S.D. Stranks, G.E. Eperon, G. Grancini, C. Menelaou, M.J.P. Alcocer, T. Leijtens, L.M. Herz, A. Petrozza, H.J. Snaith, Science 342, 341 (2013).

22. Q. Dong, Y. Fang, Y. Shao, P. Mulligan, J. Qiu, L. Cao, J. Huang, Science 347, 967 (2015).

23. G.A.H. Wetzelaer, M. Scheepers, A.M. Sempere, C. Momblona, J. Ávila, H.J. Bolink, Adv. Mater. 27, 1837 (2015).

24. R. Gottesman, E. Haltzi, L. Gouda, S. Tirosh, Y. Bouhadana, A. Zaban, E. Mosconi, F. De Angelis, J. Phys. Chem. Lett. 5, 2662 (2014).

25. 0. Almora, I. Zarazua, E. Mas-Marza, I. Mora-Sero, J. Bisquert, G. GarciaBelmonte, J. Phys. Chem. Lett. 6, 1645 (2015)

26. C.S. Ponseca, T.J. Savenije, M. Abdellah, K. Zheng, A. Yartsev, T. Pascher T. Harlang, P. Chabera, T. Pullerits, A. Stepanov, J.-P.P. Wolf, V. Sundström, J. Am. Chem. Soc. 136, 5189 (2014).

27. Y. Shao, Z. Xiao, C. Bi, Y. Yuan, J. Huang, Nat. Commun. 5, 5784 (2014)

28. A. Dymshits, A. Henning, G. Segev, Y. Rosenwaks, L. Etgar, Sci. Rep. 5, 8704 (2015).

29. M. Liu, M.B. Johnston, H.J. Snaith, Nature 501, 395 (2013)

30. Q. Chen, H. Zhou, Z. Hong, S. Luo, H.-S. Duan, H.-H. Wang, Y. Liu, G. Li, Y. Yang, J. Am. Chem. Soc. 136, 622 (2014).

31. 0. Malinkiewicz, A. Yella, Y.H. Lee, G.M.M. Espallargas, M. Graetzel, M.K. Nazeeruddin, H.J. Bolink, Nat. Photonics 8, 128 (2014).

32. K. Xiao, Y. Liu, T. Qi, W. Zhang, F. Wang, J. Gao, W. Qiu, Y. Ma, G. Cui, S. Chen, X. Zhan, G. Yu, J. Qin, W. Hu, D. Zhu, J. Am. Chem. Soc. 127, 13281 (2005)

33. A. Douhal, P. Piatkowski, B. Cohen, J.F. Ramos, M.R. Di Nunzio, M. Nazeeruddin, M. Grätzel, S. Ahmad, Phys. Chem. Chem. Phys. 17 (22), 14674 (2015).

34. Y. H. Lee, J. Luo, R. Humphry-Baker, P. Gao, M. Graetzel, M.K. Nazeeruddin Adv. Funct. Mater. (2015); doi: 10.1002/adfm.201501024.

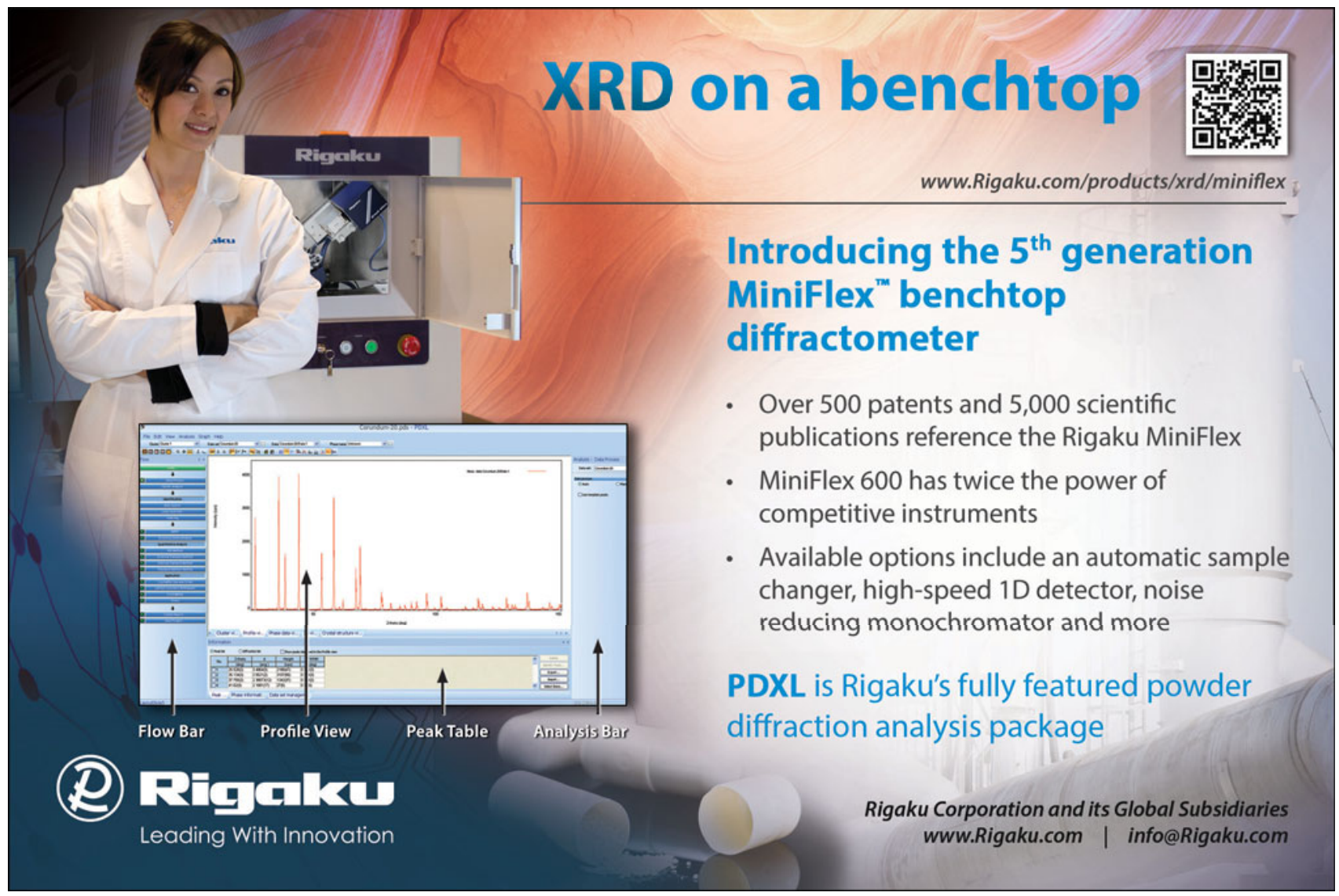

